Coitero et al. ${ }^{4}$ suggested that, in one of their cases, repeated basilar artery pulsations over a thinned bone structure could have produced the defect. Similar explanation could eventually be suggested for this particular case, considering in addition the associated condition of poorly controlled arterial blood pressure.
Advances in technological imaging tools have provided better facilities for the identification of CSF leaking sites, whereas improvements in surgical techniques, including the introduction of skull base endoscopy, have led to better surgical results. ${ }^{3}$. However, attempts to explain the pathophysiology of CSF clival fistulae remain speculative.

\title{
References
}

1. Ommaya AK. Cerebrospinal fluid rhinorrhea. Neurology 1964;14:106-113.

2. Telera S, Conte A, Cristalli G, Occhipinti E, Pompili A. Spontaneous cerebrospinal fluid rhinorrhea as the presenting symptom of sellar pathologies: three demonstrative cases. Neurosurg Rev 2007;30:78-82.

3. Elrahman HA, Malinvaud D, Bonfils NA, Daoud R, Mimoun M, Bonfils P. Endoscopic management of idiopathic spontaneous skull base fistula through the clivus. Arch Otolaryngol Head Neck Surg 2009;135:311-315.
4. Coiteiro D, Tavora L, Antunes JL. Spontaneous cerebrospinal fluid fistula through the clivus: report of two cases. Neurosurgery 1995;37:826-828.
5. Ommaya AK, DiChiro G, Baldwin M, Pennybacker JB. Nontraumatic cerebrospinal fluid rhinorrhea. J Neurol Neurosurg Psychiatry 1968;31:214-225

\section{A patient with primary progressive aphasia developing dementia due to Alzheimer's disease}

\section{Uma paciente com afasia progressiva primária devido à doença de Alzheimer}

Thais Stvan Vaz, Paulo Henrique Ferreira Bertolucci, Fabricio Ferreira de Oliveira

Department of Neurology and Neurosurgery, Escola Paulista de Medicina, Federal University of São Paulo (UNIFESP), São Paulo SP, Brazil. Correspondence: Thais Stvan Vaz; Universidade Federal de São Paulo (UNIFESP); Escola Paulista de Medicina; Departamento de Neurologia e Neurocirurgia; Rua Botucatu 740;04023-900 São Paulo SP - Brasil;E-mail:tstvan@gmail.com

Conflict of interest: There is no conflict of interest to declare.

Received 03 January 2012; Received in final for 16 February 2012; Accepted 23 January 2012

Patients with primary progressive aphasia (PPA) often evolve to frontotemporal lobar degeneration (FTLD) syndromes ${ }^{1}$, but the logopenic form of PPA has been found to have Alzheimer's disease $(\mathrm{AD})$ pathology and in vivo biomarkers suggestive of $\mathrm{AD}^{2}$. We have reported the case of a female right-handed patient with four years of schooling, who first sought assistance at the age of 63 years-old for impairments in her speaking fluency. Her daughter mentioned that two years earlier the patient began to mumble, struggling to complete sentences, often saying things out of context, with clear perception that she meant to say something else. Semantic defects were often present, when asking for a spoon and pointing to a fork, or changing the names of her children - though she knew who they were. In the previous six months, the patient progressively lost the ability to read, currently writing with difficulty. Lately, she began to show difficulties locating herself in space. Most household tasks were effortlessly performed, but she could no longer answer the phone because she could not communicate or take notes, and neither shop, since her calculating skills had been lost.
Neurological examination was largely normal. The neuropsychiatric testing displayed the following results: a 12item neuropsychiatric inventory score of 17 (mostly for anxiety and sleep disorders), with a score of 8 for caregiver distress; Clinical Dementia Rating of 1.0; score of 9 on the Mini-Mental State Examination (orientation to time $2 / 5$; orientation to space $-3 / 5$; immediate memory $-1 / 3$; calculations $-0 / 5$; recent memory $-0 / 3$; language $-3 / 9$ ); score of 1 on the 15-point Clock Drawing Test; she was able to complete one item of the Rey-Osterrieth Complex Figure Test, but was not able to recall it; score of 3 on the Boston Naming Test (15-item short version); abstract thinking was seriously impaired, while the patient was not able to read simple sentences, and scored 2 for animals and 4 for fruits in one minute for verbal fluency tests. She was independent for activities of daily living (ADL), whereas partially independent for instrumental ADL. Magnetic resonance (MR) studies of the brain showed a pattern compatible with both PPA and AD (Fig 1 and 2). 
Difficulties with language constitute the most prominent clinical feature of PPA, while cognition usually remains unimpaired at first ${ }^{1}$. Our patient has deficits in language, memory, praxis, and gnosis. This clinical picture is more likely compatible with $\mathrm{AD}$, since there is global cognitive impairment and there are no behavioral changes typical of FTLD. Even though histopathologic evidence is unavailable, clinical data point to the possibility that this patient initially presented with the logopenic variant of PPA, showing anomic hesitant speech, impaired word retrieval, and sentence repetition deficits

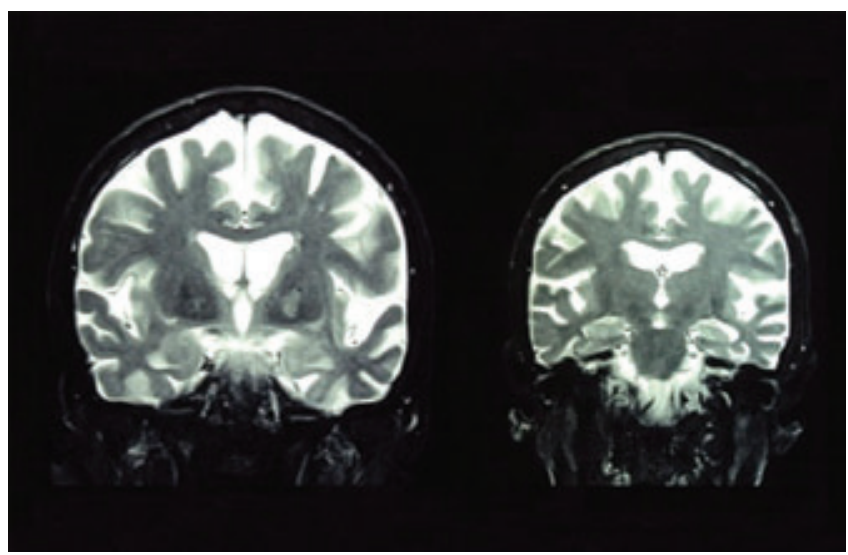

Fig 1. Coronal T2 magnetic resonance of the brain, showing global atrophy predominating in the left hippocampal and Perisylvian structures. among its core features (Table) ${ }^{1}$. This variant is present in up to $30 \%$ of patients with PPA, and it usually progresses to $\mathrm{AD}^{1,3}$. However, features that differentiate the PPA variants in the early stages may lose their distinctiveness as the degeneration advances ${ }^{4}$.

Language impairment may be the initial feature of $\mathrm{AD}^{5}$, while PPA may evolve to several dementia syndromes. This report emphasizes the need of recognizing not only FTLD and semantic dementia, but also dementia due to $\mathrm{AD}$ as a result of the evolution of PPA syndromes.

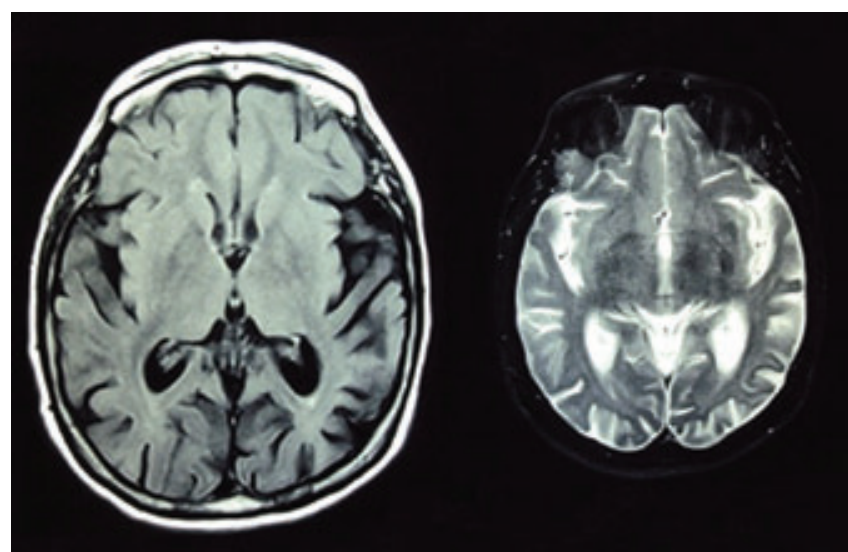

Fig 2. Axial magnetic resonance of the brain, showing global atrophy predominating in the left Perisylvian structures with no significant subcortical pathology.

Table. Diagnostic criteria for the logopenic variant of the primary progressive aphasia.

Clinical diagnosis of the logopenic variant of primary progressive aphasia

Presence of both of the following core features

A. Impaired single-word retrieval in spontaneous speech and naming;

B. Impaired repetition of sentences.

At least three of the following other features must be present:

A. Phonologic errors in spontaneous speech and naming;

B. Spared single-word comprehension and object knowledge;

C. Spared motor speech;

D. Absence of frank agrammatism.

Imaging-supported diagnosis of the logopenic variant of primary progressive aphasia

Presence of both of the following criteria:

A. Clinical diagnosis of the logopenic variant of primary progressive aphasia;

B. Imaging must show at least one of the following results:

B1. Predominant left posterior Perisylvian or parietal atrophy on the magnetic resonance;

B2. Predominant left posterior Perisylvian or parietal hypoperfusion or hypometabolism on SPECT or PET.

Logopenic variant of primary progressive aphasia with definite pathology

Clinical diagnosis (criterion A below) and either criterion B or C must be present:

A. Clinical diagnosis of the logopenic variant of primary progressive aphasia;

B. Histopathologic evidence of a specific neurodegenerative pathology;

C. Presence of a known pathogenic mutation.

\section{References}

1. Gorno-Tempini ML, Hillis AE, Weintraub S, et al. Classification of primary progressive aphasia and its variants. Neurology 2011;76:1006-1014.

2. Deramecourt V, Lebert F, Debachy B, et al. Prediction of pathology in primary progressive language and speech disorders. Neurology 2010;74:42-49.

3. Harciarek M, Kertesz A. Primary progressive aphasias and their contribution to the contemporary knowledge about the brain-language relationship. Neuropsychol Rev 2011;21:271-287.

4. Rogalski E, Cobia D, Harrison TM, Wieneke C, Weintraub S, Mesulam MM. Progression of language decline and cortical atrophy in subtypes of primary progressive aphasia. Neurology 2011;76:1804-1810.

5. Ortiz KZ, Bertolucci PHF. Alterações de linguagem nas fases iniciais da doença de Alzheimer. Arq Neuropsiquiatr 2005;63:311-317. 